\title{
MULTI-FREQUENCY RADAR LOCATION OF THE "EFFECTIVE" SOIL MOISTURE
}

\author{
Anatoly A. Kalinkevich, Boris G. Kutuza, Vasiliy N. Marchuk, Vladimir M.Masyuk
}

Kotelnikov Institute of Radioengineering and Electronics of Russian Academy of Sciences, http://www.cplire.ru/ Moscow 125009, Russian Federation

\section{Viktor A. Plyushchev}

Radio Engineering Corporation "Vega", http://www.vega.su/

Moscow 121170, Russian Federation

kalinkevich@newmail.ru,marchuk@ms.ire.rssi.ru,masyuk77@mail.ru,mail@vega.ru

Abstract: The review of studies on subsurface sensing topsoil radar method is presented, as well as on the development of an algorithm and techniques for combined interpretation of radar images using side-scan radar systems with a synthesized antenna operating over a wide range of wavelengths, including decimeter and meter bands. A method for constructing a deep profile of the "effective" moisture of the upper layer of the earth's surface is proposed. Ground experiments were conducted to determine the characteristics of the " diversity " of moisture and soil cover heterogeneities. The special attention was focused to using of ground penetrating radar for investigations of upper ground layer heterogeneities. The ground experiments were dictated by the need to justify the proposed algorithm for deep sounding of agricultural fields in the winter period at a low temperature with the help a synthetic antenna radar.

Keywords: synthetic antenna radar, ground penetrating radar, subsurface sensing, deep profile of the "effective" moisture, the "variety" of soil cover.

UDC 621.371

Bibliography - 32 references

Received 27.08.2018

RENSIT, 2018, 10(2):193-208

DOI: $10.17725 /$ rensit.2018.10.193

\section{CONTENT}

1. INTRODUCTION (193)

2. Substantiation OF THE APPROACH FOR DETERMINATION OF THE DEEP SURFACE (195)

3. EXPERIMENTAL INVESTIGATIONS OF SOIL COVER (197) 3.1. Prerequisites for CONDUCting EXPERIMENTAL STUDIES (197)

3.2. EXPERIMENT TO MEASURE THE "VARIETY" OF THE MOISTURE CONTENT AT DIFFERENT DEPTHS OF THE UPPER SOIL LAYER (198)

3.3. "VARIETY" OF THE SUBSURFACE LAYER OF THE EARTH'S SURFACE (199)

4. Construction of the Depth profile of "EFFective" humidity By Results of MULTIFREQUENCY RADAR LOCATION (202)

5. EXPERIMENTAL RESUlts (204)

5.1. BUILDING AN EFFECTIVE MOISTURE PROFILE FROM DEPTH (204)

5.2. ANALYSIS OF THE OBTAINED INTERPRETATION RESUlTs (205)

6. Conclusion (206)

REFERENCES (206)

\section{INTRODUCTION}

In a number of countries have developed aircraft synthetic aperture radar $(S A R)$ operating in wide range of wavelengths, including decimeter $(P)$ and meter $(V H F)$ bands [1-5]. Comparison of radar images of the same area in different wavelengths shows the qualitative differences that are connected with the features of the surface reflection and the influence of reflection and absorption of electromagnetic radiation from subsurface layers. Electromagnetic radiation of different wavelengths penetrates into the land cover at different depths, so the question of the development of methods of using radar location images (RLI) obtained in different wavelength ranges for deep layered sensing of earth covers raises. This method of layered subsurface sensing qualitatively different from the classical method based on the sensing of short pulses.

In Russia the complex SAR "IMARC" [1] operating simultaneously in four wavelength ranges $X(4 \mathrm{~cm}), L(23 \mathrm{~cm}), P(68 \mathrm{~cm})$ and $\operatorname{VHF}(254 \mathrm{~cm})$ at various polarizations $H H, V V, V H$ and $H V$ is 
developed. This complex gave the possibility to obtain multi-frequency polarization matrix of radar images. Analysis of radar images of the complex allowed us to offer one of the possible methods for constructing depth profiles of the "effective" soil moisture of agricultural lands in winter time in the European part of Russia. Ground-based experiments for mapping of soil moisture at different depths in limited areas and the results of determination of parameters of inhomogeneities in the upper subsurface layers with GPR was dictated by the need to justify the proposed algorithm for joint interpretation of radar images of the same area obtained in different wavelength ranges.

Until recently the real practice of subsurface sensing was related to the time of separation of signals coming from subsurface layers and objects located below the surface. A subsurface remote sensing from the airplane (helicopter) or the surface of layered earth cover by radio impulse $[6,7]$ is considered to be generally accepted. The effective duration of these impulses is nanoseconds and less at the normal direction of propagation of electromagnetic radiation to the surface.

Reflection occurs at the junction of layers or objects having different dielectric properties. The interpretation is based on the separation of signals over time from the surface of various layers or objects located under the soil layers. The method of probing the Earth's surface using vertical sensing at different frequencies is much less frequently used $[8,16]$. Despite the complex subsurface structure of the Earth cover, great success has been achieved in the application of GPR for solving a number of scientific and applied problems [7].

At present, aviation complexes of SAR operating in a wide range of wavelengths, including decimeter $(P)$ and meter (VHF) ranges [1-5] have been developed in a number of countries. Comparison of radar images $(R I)$ of the same terrain in different wave bands shows qualitative differences that are related both to the features of surface reflection and to the effect of reflection and absorption of electromagnetic radiation from subsurface layers. Electromagnetic radiation (EM) of different wavelengths penetrates into cover at different depths, so the question of the development of methods for the use of RLI in different wavelength ranges for deep layer-by-layer sensing of earth cover.
This method of layer-by-layer subsurface sounding is qualitatively different from the classical method based on sensing the earth's surface with short pulses.

In normal sensing with the help of GPR the analysis uses mirror or quasi-mirror reflection components in combination with the laws of reflection and refraction. Diffuse components are a side effect that makes interpretation difficult. Radar images correspond to the diffuse scattering component, which is related to the roughness, inhomogeneities of the upper layer of the earth's surface. Simulation of the back reflection for different EM wavelengths from the unknown structure of the inhomogeneities of the subsurface layer is impossible in general. In the simplest cases, the role of reflection from the subsurface layers is attempted to be taken into account by their influence on the reflective properties of the surface layer. For example, the physical basis for the study (detection) of groundwater is the change in the back reflection on the roughness of the soil cover caused by additional moistening of the upper layers of the soil due to capillary phenomena - by changing the moisture of the overlying soil layers (capillary rim) and, consequently, reflectivity. Despite the attractiveness of the idea of using multi-frequency SAR for constructing a deep profile of the upper layer of the Earth cover, there is no noticeable breakthrough in this direction. Therefore, consideration of any attempts to jointly use multi-frequency radar images to determine the depth profile of the upper layer of the earth's surface is of great interest.

In this paper, we consider the possibility of constructing a deep profile of a homogeneous part of the earth's surface based on the results obtained with the help of the SAR that was developed by the Concern "Vega" [1]. The complex simultaneously operated in four wavelengths $4 \mathrm{~cm}, 23 \mathrm{~cm}, 68 \mathrm{~cm}$ and $254 \mathrm{~cm}$ in different polarizations $H H, V V, V H$ and $H V$. The analysis of the RLI of this complex made it possible to propose one of the possible methods for constructing a depth profile of the "effective" (see below) soil moisture of agricultural lands in the winter period in the European part of Russia.

Ground experiments on soil moisture mapping at different depths in some parts of the terrain, as well as to determine the parameters of inhomogeneities in the upper subsurface layers with the help of GPR 
were dictated by the necessity to substantiate the proposed algorithm for the joint interpretation of the same radar plot obtained in different wavelength ranges.

When considering the problem, two principal questions arose:

1. How can we perform the modeling of the upper layer of the underlying surface with an unknown structure to build a deep profile?

2. We must develop an algorithm for calculating the depth profile based on the results of radar survey in different wavelength ranges.

\section{SUBSTANTIATION OF THE APPROACH FOR DETERMINATION OF THE DEEP SURFACE}

The first step in solving the problems of subsurface sensing of soil cover with the help of multifrequency SAR was the calculation of the estimates of the backscattering signal from different subsurface objects at wavelengths 4, 23, 68 and $254 \mathrm{~cm}$ which are used in the SAR complex [1]. The calculations were performed under the assumption that there is no surface roughness and volume scattering but only taking into account the attenuation in the medium. The results of model calculations of scattering from a spherical object, a dielectric disk, a metal plate in a soil are given in $[9-13,15]$. In this case, the problem of finding reflection from a dielectric object was solved in a rigorous electro-dynamic formulation. The dependence of the effective scattering area (ESA) on the frequency of the signal, on the size of the object, on the parameters of the soil, on the direction of incidence of the plane wave, and on the polarization was investigated. For calculating the absorption in wet sandy and clayey soils wet sandy the model presented in $[9,14]$ was used. As a result of calculations in $[12,13]$ the following conclusions were made.

1. For a dielectric object or a cavity in the ground, when the size of the object is comparable to the wavelength, oscillations of the reflected signal are observed depending on the wavelength in consequence of the resonance effects. For large wavelengths, the signal decreases monotonically with increasing wavelength.

2. For extended objects at high frequencies, oscillations of the reflected signal are observed as a function of the angle of incidence which is due to the rupture of the backscattering diagram.

3. The amplitude of the reflected signal can serve as one of the signs on the basis of which, when using a set of frequencies, it is possible to make assumptions about the nature of the object found in the ground.

4. The dependence of the amplitude of the reflected signal on the wavelength and the angle of incidence, in principle, can be used to estimate the dimensions of subsurface objects. However the absence of a priori information on the depth of the location of the object and the properties of the soil, the strong dependence of the attenuation on the wavelength, are a serious obstacle in the recognition of objects.

5. The recognition problem can be posed for objects located in sandy ground or sandy-clay soil with a low clay content and low moisture.

6. Losses are very large in the clayey soil, so that sensing is possible only to very small depths less than $0.5 \mathrm{~m}$ when long waves are used.

In accordance with conclusions 4-6, you can "outline" the range of tasks that can be addressed:

a) subsurface remote sensing with a relatively simple geological structure and with a special dryness of rocks in the absence of vegetation cover;

b) subsurface remote sensing of frozen soils;

c) solving problems such as mapping the hydrological regime when the groundwater depth is located at a shallow depth (mapping of flood zones).

Similar calculations and estimates of scattering of objects can be found in other works.

In the solution of the reflection problem even in a rigorous electro-dynamic formulation the surface layer above the object is assumed to be homogeneous with known dielectric properties, scattering from the soil layer is not taken into account (only attenuation is taken into account), the reflection from the object and the soil layer are not compared.

In addition, based on calculations for reflection from various objects in homogeneous soils, carried out in these studies, the following conclusions were drawn.

The dependence of the amplitude of the reflected signal on the wavelength, in principle, can be used for the purpose of identifying subsurface objects. The amplitude of the reflected signal can serve as one of the indications on the basis of which, when using a 
set of frequencies, it is possible to make assumptions about the nature of objects in the ground.

Apparently, the recognition problem can be posed for objects located in sandy ground or sandyargillaceous ground with low clay content or low moisture. Losses are very high in clay so that remote sensing is possible only to very shallow depths - less than $0.5 \mathrm{~m}$ or in the case of relatively low losses (low temperature, low moisture) up to 1-1.5 $\mathrm{m}$. The losses are especially high at high frequencies. Therefore, the longest waves $254 \mathrm{~cm}$, and in some cases $68 \mathrm{~cm}$ can practically be used from the existing set of having wavelengths. The insufficient number of frequencies at which a signal can be received and analyzed is an obstacle in the task of identifying objects.

In addition, in the absence of a priori information on the depth of the location of the object and the strong dependence of the attenuation on the wavelength, the effects associated with the influence of the shape and type of the object on the amplitude of the reflected signal will be greatly distorted by the effect of attenuation in the ground.

Oscillations of the reflected signal are observed depending on the wavelength due to resonance effects when we investigate a dielectric object or cavity in the ground at sufficiently high frequencies and when the size of the object is comparable with the wavelength. For big wavelengths the signal decreases monotonically with increasing wave length.

For extended metal objects at high frequencies oscillations of the reflected signal are observed depending on the angle of incidence. The oscillations are due to the rupture of the scattering diagram. The ruggedness of the scattering diagram can serve to estimate the size of a metal object. However, this requires a larger set of wavelengths with a smaller difference in neighboring wavelengths than in the considered wavelength set. The largest signal in the case of high frequencies is expected within the narrow lobes of the scattering diagram. At low frequencies, the reflected signal is much larger at large angles of incidence far from the normal to the surface of the object. In the case of extended dielectric objects, the discontinuity of the scattering diagram can be less pronounced.

The amplitude of the reflected signal decreases strongly when the sensing direction approaches to the horizon. Therefore it is necessary to choose the sensing angles the closest to the normal.
As a consequence of these conclusions the $X$ and $C$ bands have limited possibilities for subsurface remote sensing. The key moment for the development of the technique and algorithm for layer-by-layer subsurface remote sensing will be the representation of the volume inverse reflection mechanism.

However the main drawback of the performed computations is that the object is considered to be located in a homogeneous medium whose surface is flat.

The complexity of using multi-frequency polarization images for layered subsurface remote sensing of vegetation and soil cover is that it is necessary to use volumetric scattering information, which is practically nonexistent. Taking into account the fact that the composition, structure, mineralization and moisture of the subsurface layers can be very diverse and affect the reflection in different wavelength ranges is different, a detailed modeling of the backscattering from the subsurface layers and objects is very problematic even in each individual case.

Under studying reflection from the earth's surface with oblique sensing the focus is on reflection from the rough boundary of the surface, assuming that the surface has constant dielectric properties. The backscattering for oblique sensing is determined by the roughness of the section - the ratio of the root-mean-square deviation of the surface height $(\sigma)$ and the wavelength of the radio emission $(\lambda)$ $\sigma / \lambda$. In the case of a 4-frequency SAR, the ratios $\sigma /$ $\lambda_{i}$ can have a wide spread: $\sigma / \lambda>>1$ for the shortwavelength range $\lambda=4 \mathrm{~cm} ; \sigma / \lambda<<1$ for $\mathrm{P}(\lambda=68$ $\mathrm{cm})$ and $\operatorname{VHF}(\lambda=2.54 \mathrm{~m})$ ranges. The surface can be considered smooth if $\sigma<\lambda / 2 \cos \Theta$ (Rayleigh criterion). According to the above criterion for a long-wavelength SAR channels a number of surfaces are smooth.

In $[20,21]$, an approach (the method of small perturbations $\sigma / \lambda<<1$ ) is considered for solving scattering on slightly rough surfaces. As a result, the following conclusions are drawn:

1) The backscattering for $\mathrm{VV}$ and $\mathrm{HH}$ polarizations depends on the difference in the dielectric properties of the air and the surface $\sim|\Delta \varepsilon|^{2}$, and also on the spectral density of the surface roughness under the condition $2 \pi / \Lambda=2(2 \pi / \lambda) \sin \Theta$, where $\lambda$ is the wavelength of the electromagnetic radiation, $\Delta$ - the 
roughness wavelength, and $\Theta$ - the observation angle (Bragg condition).

2)The cross-polarization components of the scattered field are equal to zero.

Radar images of the earth's surface at wavelengths of $4,123,68,254 \mathrm{~cm}$ have shown that the crosspolarization components of the scattered field are always observed, even for the VHF range, when many slightly rough surfaces can be considered smooth.

It should be noted that in the presence of vegetation cover the problem of reverse reflection is greatly complicated. Because of the complexity of modeling surface irregularities, especially with vegetation and inhomogeneities in the upper soil layer. The upper layer of the Earth in modeling in a number of cases is represented by a planar layered structure whose layers have different dielectric properties. However, when considering the upper layer of the Earth's surface in the form of homogeneous dielectric layers having smooth boundaries, back reflection (according to the laws of geometric optics) should be absent, which does not correspond to the experiments.

In [22-24], the question of effect of soil moisture on back reflection was investigated (in some experiments, the surface was rolled up to reduce the roughness). It was shown that the inverse reflection at some angle to the surface is directly proportional to moisture $(m): \sigma_{0 \mathrm{i}}=L_{0 \mathrm{i}}+L_{1 \mathrm{i}} m$ and weakly depends on the surface roughness. From the obtained results, one can conclude that the inverse reflection is determined not only by the surface roughness, but also depends on the dielectric spatial inhomogeneity of the surface. In this case, the characteristics of these inhomogeneities depend on the average value of soil moisture. By analogy with the reflection from a rough surface, in order to explain the results obtained in $[22,24]$, it can be assumed that the inverse reflection depends on the value of the spectral density of the dielectric spatial inhomogeneity of the surface, i.e. from the spectral density of the spatial spectrum of the dielectric inhomogeneities of the surface under the condition $2 \pi / \Lambda=2(2 \pi / \lambda) \sin \Theta$ where $\lambda$ is the wavelength of the electromagnetic radiation, $\Lambda$ - the wavelength of the spatial dielectric inhomogeneity, and $\Theta$ - the observation angle (the Bragg condition). With such an examination, if the spatial physical properties of the surface change, then an inverse reflection of electromagnetic radiation (EMR) should also be observed from an even flat surface.

Thus, the reverse reflection for oblique sensing should depend not only on the spatial spectrum of the surface roughness, but also on fluctuations in the dielectric properties of the surface - the inverse reflection will be proportional to the Bragg spectral component of the fluctuations as the roughness of the dielectric permittivity of the surface.

The heterogeneity of the soil cover is a widely known phenomenon. In soil science there is the concept of "variety" and the individuality of the soil cover [25]. The term "variety" of the soil cover is understood to mean the heterogeneity of the properties of the soil cover along the horizontal plane, and also in depth. A review of the literature on this question can be found in [25]. Despite the extensive research on this issue, it was impossible to use the results obtained by soil scientists to develop a mechanism for the back reflection of EM waves due to the lack of specific values for both the magnitude of the fluctuations and the spatial spectral functions of these inhomogeneities. This explains the need for ground-based research on the physical characteristics of the upper surface layer.

\section{EXPERIMENTAL INVESTIGATIONS OF THE SOIL COVER OF THE SOIL COVER}

3.1. PREREQUiSITES FOR CONDUCTING EXPERIMENTAL STUDIES

It is known that moisture first of all determines the dielectric properties of the soil (sand, loam, clay, etc.) [9, 14]. With the increase in soil moisture, the filling of micro pores with water increases, the uneven filling of micro pores leads to an increase in the spatial inhomogeneity of the dielectric constant. Confirmation of this can be observed by analyzing the results of experimental works [25]: when drilling a borehole over a water lens in the desert, it turned out that with increasing mean moisture content of the soil its fluctuations in depth increase (Fig. 1).

The authors do not relate the fluctuations in moisture to the presence of a layered soil structure. Under modeling of back reflection, when the upper layer of the Earth's surface is represented in the form of flat layers, only the spectral density of the "variety" of the permittivity must be taken into account. 


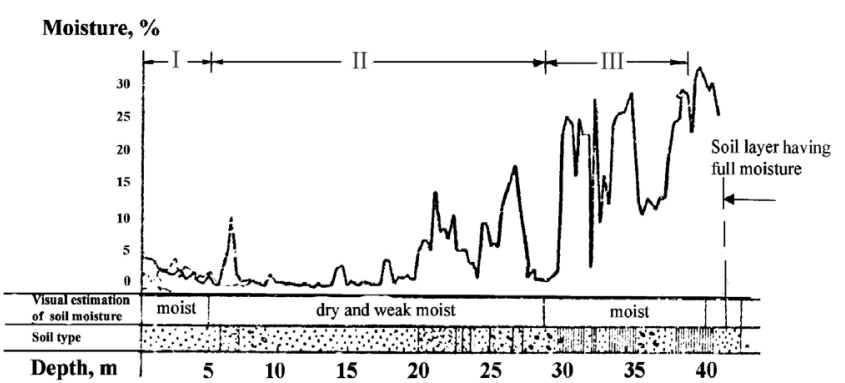

Fig. 1. Diagrams of moisture in the zone of fixed sand in the Kara-Kum Desert.

Extensive studies [14] of the influence of sand moisture on the absorbing properties for different wavelengths were made (the moisture value allows us to calculate the attenuation for the layer).

In our work, all the changes in the permittivity in a flat sub-layer are correlated to the "variety" of the moisture content of the sand. This is one of the assumptions of our model for the subsequent construction of a deep profile of the upper soil (changes in soil heterogeneity, roughness, salinity, etc., we correlate to the moisture content of sand). In this case, at first glance, unexpectedly very large and small (unreal) moisture values may appear, which we referred to as "effective" moisture).

This is due to the fact that we do not know anything about the structure of the subsurface layer. This is the main postulate of our work, which can be disputed. As a result, as will be shown below, the resulting deep profile of "effective" moisture requires additional physical interpretation, i.e. it is necessary to relate this or that layer of "effective" moisture to a tangible physical value, for example, snow cover, soil roughness under snow cover, frozen soil, etc. This stage of work is performed from purely a priori information, which must correspond to the physical meaning.

Fig. 2 shows a diagram of the layers of the Earth's surface involved in the formation of the back reflection.

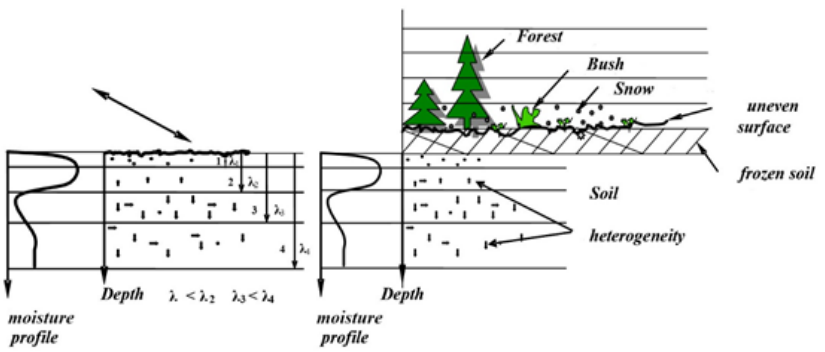

Fig. 2. Scheme of distribution of soil layers participating in the reverse reflection of the radar signal of different wavelength ranges.
In the absence of vegetation the RLI in the $4 \mathrm{~cm}$ range is formed by the layer 1 , which may include roughness, in the $23 \mathrm{~cm}$ range - by the soil layer 2, also including the surface roughness, and so on.

\subsection{EXPERIMENT TO MEASURE THE "VARIETY" OF} THE MOISTURE CONTENT AT DIFFERENT DEPTHS OF THE UPPER SOIL LAYER

Experimental research was carried out in the autumn, when the green grass cover was absent. Along three parallel lines, located at a distance of $50 \mathrm{~cm}$ from each other and 7 meters long on a flat horizontal surface every $50 \mathrm{~cm}$, boreholes were drilled to a depth of $2 \mathrm{~m}$. From the depths of 30 $\mathrm{cm}, 83 \mathrm{~cm}, 136 \mathrm{~cm}, 189 \mathrm{~cm}$ soil samples were taken to further determine the moisture content. Analysis of the samples showed that the upper layer of the soil is clay silt, to the depth of $83 \mathrm{~cm}$ - the average loam, at depths of $136 \mathrm{~cm}$ and $189 \mathrm{~cm}$ - moraine moss, i.e. the soil composition was relatively homogeneous. Fig. 3 shows the dependence of soil moisture on the distance along one of the straight lines corresponding to different depths. The length of the plot of 7 meters showed variations in soil moisture. Measurements of moisture in soil layers located at different depths from the surface showed that the soil moisture fluctuates for each depth relative to the average value at least $50 \mathrm{~cm}$ apart and for different depths and practically independently of each other (correlation coefficient $R<<1.7$ ). Since in the layers located at different depths, the average humidity turned out to be different, and the soil composition was relatively homogeneous, a comparison of the dispersion value with the average moisture value showed that for a given humidity range and a particular soil section the magnitude of humidity fluctuations increases with increasing soil

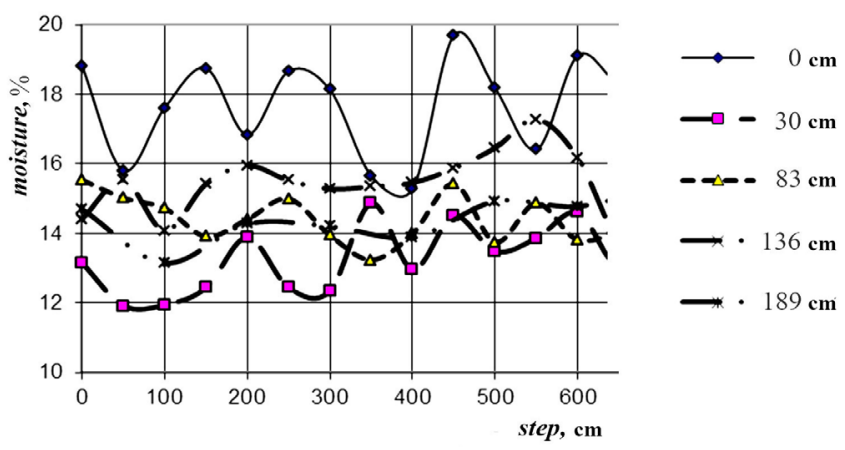

Fig. 3. An example is the spatial variation of soil moisture at different depths. 


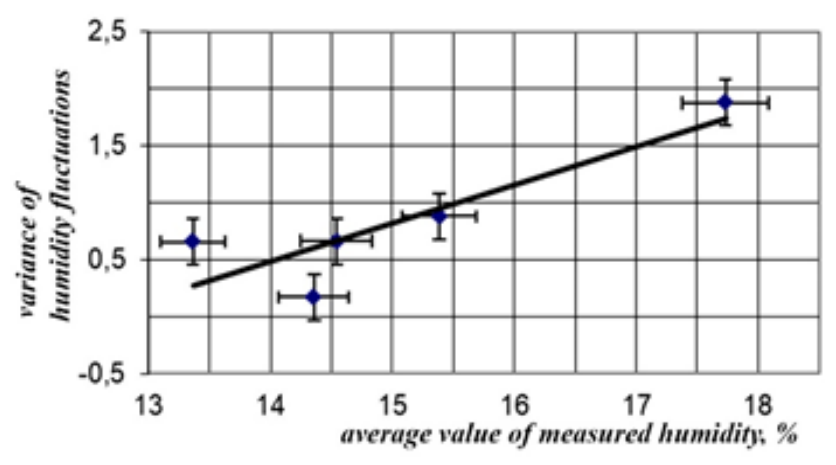

Fig. 4. Dependence of dispersion of bumidity fluctuations on average values of measured soil moisture.

moisture $\delta m_{l}=C_{1} m_{l}\left(m_{l}\right.$ is the average moisture value in layer 1 in Fig. 3).

From this experiment the following conclusions were drawn:

1) indeed, at least at a distance of $50 \mathrm{~cm}$ both in depth and in space, the humidity changes,

2) the mean square value of humidity fluctuations is directly proportional to the value of humidity (at least for the given experiment at the indicated values of humidity) Fig. 4,

3) analysis of the dependence of humidity fluctuations along a straight line at different depths showed that humidity fluctuations at different depths are slightly correlated (mean value of correlation coefficient of moisture fluctuations between layers located at a distance of $30 \mathrm{~cm}$ was 0.17 ).

The second experiment was carried out in the following way: on the area of $1 \mathrm{~m}^{2}$ every $10 \mathrm{~cm}$ from the upper layer soil samples were taken for moisture measurement.

Based on the results of these two experiments, the spatial spectra of humidity fluctuations were calculated, $1 / \mathrm{cm}$. The experimental data are shown in Fig. 5.

Characteristic was the fact that the spatial moisture spectra are broad, the spectral densities for wavelengths of 23, 68 and $254 \mathrm{~cm}$ are approximately the same, although experiments were conducted at different times in different suburban areas.
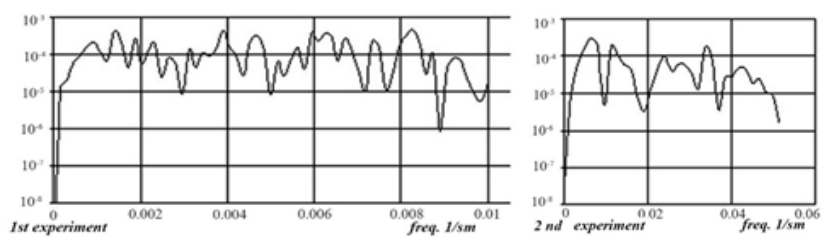

Fig. 5. Spatial moisture spectrum.

\section{3. "VARIETY" of THE SUbSURface LaYer of THE EARTH'S SURFACE}

The heterogeneity of the soil cover is determined not only by moisture, but also by soil type, salinity, roughness, the presence of the root system of plants, etc. However, drilling studies do not allow the study of such inhomogeneities.

It is known that to study layered soil systems, the detection of objects in the upper soil layers, etc. GPR are used. At the same time, there are doubts whether it is possible to use GPR to study the inhomogeneities of the medium. The attempt was made to use two types of GERADtype GPR systems $[26,27]$ to assess the nature of heterogeneities in the permittivity of the upper soil layer in two sections of an even surface which had different values of the backscattering in the VHF band. The experiment was carried out in the following conditions: in a free section at a distance of 20 meters from each other, boreholes were drilled to a depth of 2.5-3.0 $\mathrm{m}$ and a deep moisture profile was measured (Fig. 6).

In both regions the soil is sandy and to a depth of about 2.5 meters sand moisture $~ 7 \div 10 \%$ (the real part of the complex dielectric permittivity $\varepsilon \approx 8$ ), the thickness of the capillary rim is about 1 meter.

To isolate the reflected signals, determine their time shifts, etc. A wide arsenal of established methods is used [27, 28]. In this paper we used methods for processing the results of georadar surveys [27]. The optimal sequence of processing steps of GPR survey of the upper layer of the soil in the forest was worked out according to the results of measuring the reflection from a metal object buried in the earth to a depth of $70 \mathrm{~cm}$ from the surface.
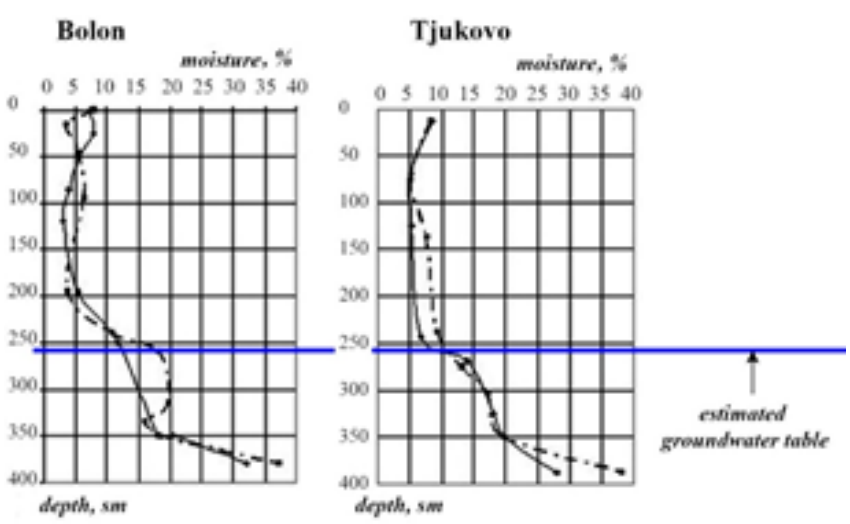

Fig. 6. Depth moisture profiles in two study areas. 
The main processing steps and their influence are shown in Fig. 7.

The image in Fig.7, No. 1 corresponds to the original image, the first stage of processing corresponds to the Hilbert transform - obtaining an image in a unipolar form and emphasizing the transition from white to black. The type of the georadar image after such processing is shown in the figure (Fig. 7, No. 2). The next stage is connected with finding the impulse response of the medium. It is known [6] that the signal reflected $u(t)$ from the surface can be represented as convolution of a radiated signal $s(t)$ with an impulse response of the medium $g(t): u(t)=s(t) * g(t)$. Since convolution in the time domain corresponds to the product of the spectra of the corresponding functions in the spectral region [28], then: to obtain the impulse response, we need to perform the operation:

$$
G(f)=U(f) / S(f)
$$

and calculate the inverse Fourier transform of $G(f)$. Here $U(f)=f(t(t)), S(f)=f(t(t)), G(f)=f t(g(t))$, where $f t$ is the Fourier transform. The problem is that the modulus of the spectrum of the original signal vanishes in some regions of the selected frequency range, as a result, the uncertainty of the type of division by zero can arise in the calculation using formula (1). To solve this problem, the authors of [28] proposed the following solution: when calculating equation (1) in the denominator, one introduces a deliberately positive additive much smaller than the maximum amplitude: $S(f): G(f)=$ $U(f) / S(f)+0.01 \max S(f)$. In this case, the impulse response of the medium will be: $g(t)=i f f t(U(f) / S(f)$ $+0.01 \max S(f))$, here ifft is the fast inverse Fourier transform. This algorithm is implemented in the

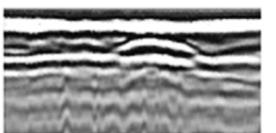

№ 1
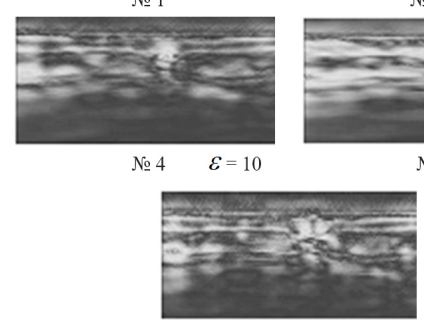

№ $7 \quad \varepsilon=20$

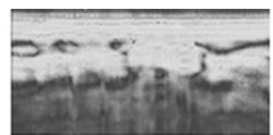

№ 2

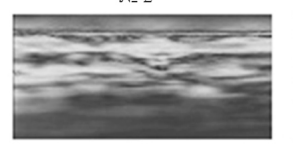

№ $5 \varepsilon=5$

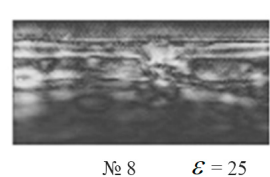

Fig. 7. Elaboration of a technique for processing GPR survey on a metal object located in the upper layer of sand for different values of the permittivity. inverse filtering procedure in this paper. After this processing step, the GPR image of the metal object looks as shown in Fig. 7 (No. 3).

It should be emphasized that all restrictions related to spectral processing (distortion of the pulse due to dispersion and the frequency dependence of the absorption) are no less, and may even be more relevant to inverse filtering. That is, reliable results using inverse filtering can be expected in nonabsorbing media, or in weakly absorbing media at relatively shallow depths. Note: in [29], inverse filtering is seriously criticized. However, this method turned out to be one of the main stages of processing in this paper.

The final stage of processing corresponded to the performance of synthesizing the aperture in the direction of movement of the antenna block for various values of the dielectric constant. The synthesis of the image can be carried out at different values of the permittivity, which characterizes the propagation velocity of EM waves in the medium. Fig. 7 (№ $4 \div 8)$ presents processed GPR images with different values $\varepsilon$. The analysis of the obtained images showed that the optimum image is obtained at $\varepsilon=10$. As one was noted earlier, when drilling wells and measuring moisture profiles, the real part of the dielectric constant of the upper soil layer turned out to be equal $\varepsilon \approx 8$. Values $\varepsilon$ obtained in different ways are consistent. Later, when processing the measurements, we used $\varepsilon=10$.

The GPR survey was carried out along the line between the drilling points and along lines parallel to it at distances of 0.5 and 1 meter. To account for the fluctuations in motion, a pair of GPR surveys were conducted along each line. To compare the results of different "passes" (as far as the images of the two passes differ, coincide with each other), a physicomathematical value was introduced [30] - the average value of the coefficient of autocorrelations between different layers. Its meaning is presented below. In Fig. 8 the results of GPR survey are shown in two sections of the surface located one from another at a distance of about $5 \mathrm{~km}$. The drilling points $A, B$, $C, D$ and the effect of drilling on the nearby soil are shown in the Fig. 8.

On GPR images, the upper soil layer (up to $2.5 \mathrm{~m}$ ) was split into a number of sublevels (the quantization step $\sim$ corresponded to the range resolution element). For each sublevel of the received GPR images, the 


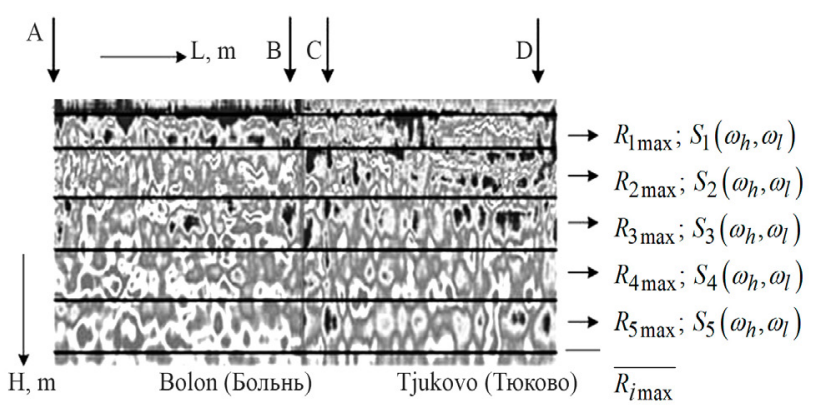

Fig. 8. Results of GPR survey in two parts of the surface between the drilling points.

mutual autocorrelation function and its maximum value $R_{1 \mathrm{~m}}$ were calculated. The average value of the maximum values of the correlation coefficients $\bar{R}_{\mathrm{Imax}}$ was used as a quantitative measure of the coincidence of the survey results. The processing of GPR results by this method for the same site allowed us to estimate by the values $\bar{R}_{1 \max }$ the repeatability of the results of each "pass", and also at what offset aside from the central line of the "passage" the correlation disappears. As an example, Table 1 gives the values of the maximum correlation coefficients for two passes along a single line

The results of the processing of graphic files showed that the correlation analysis is very sensitive to the accuracy of "setting" the initial parameters - it is necessary to set very carefully the beginning of scanning horizontally and vertically. In addition, there is a strong dependence on the contrast of images. Hence it follows that during processing it would be desirable to introduce one more step preliminary filtration of secondary images, especially in terms of increasing contrast and reducing noise.

The secondary images obtained after the treatment considered above also made it possible to calculate the spatial spectrum of inhomogeneities at different depths. In Fig. 9 shows the spatial spectrum of inhomogeneities at different depths of two surface sections.

Table 1.

Correlation values for repeated passes.

\begin{tabular}{|c|c|}
\hline Plot of land & Correlation coefficient \\
\hline R1 & 0.4061 \\
\hline R2 & 0.5839 \\
\hline R3 & 0.3058 \\
\hline R4 & 0.5183 \\
\hline R5 & 0.5111 \\
\hline$R_{\text {срев }}$ & 0.4650 \\
\hline
\end{tabular}
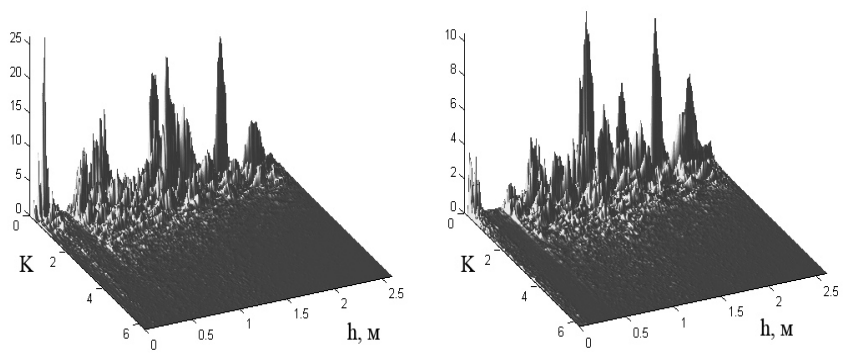

Fig. 9. Spectrum of spatial fluctuations of inhomogeneities for two sections.

In Fig. 9 the $x$ axis represents the spatial wave number $K$, the $y$ axis the depth, and $z$ the intensity of the spectrum in relative units. It can be seen that the spatial fluctuations of the inhomogeneities, measured with the help of GPR, are concentrated at depths of up to one meter (up to the penetration depths of the EMR) and have a very rugged appearance. In addition, spatial spectra along parallel lines spaced $0.5 \mathrm{~m}$ apart are independent (the mutual correlation coefficient was about 0.1). When averaging over three lines parallel to spaced apart distances of 0.5 and 1 meter, the spatial spectra are smoothed out.

\section{CONSTRUCTION OF THE DEPTH PROFILE OF "EFFECTIVE" HUMIDITY BY RESULTS OF MULTIFREQUENCY RADAR LOCATION}

The task of remote sensing of terrestrial coverings by means of radio signals in different wavelength ranges is related to the determination of the distribution profile of the complex permittivity of the investigated surface $\varepsilon(z)$, where $z$ is the coordinate associated with the depth.

Interpretation of radar images of terrestrial coverings at different frequencies represents an incorrect inverse problem $[8,19,31]$. An approximate solution of the inverse problem in the general case is found by constructing a smoothing functional representing the discrepancy functional between the results of measurements of the scattered field and the model direct problem [31]. This task in the general case consists in the determination of the required parameters by the informative characteristic $P(\varepsilon)$ obtained from the reflected signal. One of the most universal informative parameters of the investigated object is the frequency dependence of the complex reflection coefficient. As indicated in [30], a popular approach to solving similar problems is the method of parametric optimization, which 
consists in constructing a mathematical model of the investigated medium and minimizing the functional (objective function) $\rho(\varepsilon)$ characterizing the distance between the informative parameter measured experimentally $P_{\mathrm{e}}(f, \varepsilon)$ and theoretically calculated $P_{\mathrm{t}}(f, \varepsilon)$. The objective function is defined as follows

$$
\rho(\varepsilon)=\sum_{i=1}^{L}\left|\frac{P_{e}\left(f_{i}, \varepsilon\right)-P_{t}\left(f_{i}, \varepsilon\right)}{P_{t}\left(f_{i}, \varepsilon\right)}\right|^{2},
$$

where $f_{\mathrm{i}}(i=1,2, \ldots, L)$ is the set of frequencies at which the informative parameters are measured and calculated.

According to [31], the inverse problem has a unique and stable solution only if the initial experimental and calculated theoretical data are determined on an infinite frequency interval, which in practice is unattainable. An experimentally informative parameter is measured with some error. These circumstances cause incorrectness of the inverse problem being solved, which is expressed in the existence of a large number of side minima of the functional (2). In addition, in order to calculate the model information sign $P_{\mathrm{t}}(f, \varepsilon)$, a direct electrodynamic problem must be solved, which is also a problem. To improve the solution of such an illposed problem, various algorithms are used: the neural method [32], the use of additional a priori information in constructing a smoothing functional [8].

In the case of using the SAR IMARK complex for the purpose of subsurface sounding, the problem is complicated by the fact that only 4 wavelengths are used, as well as the impossibility of creating even an approximate model of the inverse reflection of the subsurface volume. Under such conditions, the main attention should be paid to a priori information. In general, the search for solutions will be limited to curves (the moisture profile in depth), which can be approximated by polynomials of the third degree.

To develop an algorithm for layer-by-layer sounding of terrestrial surfaces, a direct electrodynamic problem must be solved. To implement the method of constructing a depth profile, as a first assumption, the surface is represented in the form of flat thin parallel sub-layers, regardless of the type (plant, snow cover, frozen soil, etc.). Each sub-layer is characterized by a coefficient of attenuation and reflection. Let's imagine that there is a surface area with a homogeneous reflectivity $\sigma_{0 \mathrm{i}}$ (the effective scattering area for four wavelengths $\lambda_{i}$ is different). In this case $\sigma_{0 \mathrm{i}}$ - the effective coefficient of back reflection of a thin layer at the depth of occurrence $b$ when sounding at an angle $\theta$. Then the EPR $\sigma_{0 \mathrm{i}}$ value is represented as:

$$
\sigma_{0 i}(\theta)=A \int_{0}^{\infty} \sigma_{i}(h) e^{-2 \int_{0}^{\cos \theta_{i}} \alpha_{i}(x) d x} d h,
$$

where $\alpha_{i}(b)$ is the dependence of the attenuation coefficient of electromagnetic waves for the wavelength $\lambda_{i}$ on the depth, $\theta$ is the angle of propagation of electromagnetic waves (at this stage it is assumed that it is constant in the medium). It is seen that it is necessary to simulate two dependencies $\alpha_{i}(b)$ and $\sigma_{i}(b)$. The back reflection of a thin layer from the depth of occurrence $b$ and its possible connection with the attention coefficient $\alpha_{i}(b)$ are unknown. The reverse reflection in the case of a planar sub-layer will be observed only in the presence of spatial dielectric inhomogeneities in the su-layer itself within the radar resolution element. Thus, it is necessary to relate the attenuation coefficient to the reflection coefficient. Initially, we will perform this procedure for a moist, homogenous soil, having a dependence $\alpha_{i}(b)$ - the attenuation coefficient on depth with the dependence $\sigma_{i}(b)$ - the reflection coefficient for different wavelengths.

Numerous experimental data have shown that the inverse reflection at some angle to the surface is directly proportional to moisture: and depends little on the surface roughness (see Fig. 10).

The increase in humidity leads both to an increase in the attenuation coefficient, and to an increase in the reverse reflection. For a homogeneous layer of soil with a constant moisture content in depth, following expression (3), the reflection coefficient must have the form. Suppose that there is a relationship between the attenuation and reflection coefficients of a thin layer of the type.

$\sigma_{i}(b)=\beta_{0 i} \alpha_{i}(b)+\beta_{1 i} \alpha_{i}^{2}(b)$.

Since $\alpha \sim m$ then following Eq. (3), the EPR will indeed have the form $\sigma_{0 \mathrm{i}}=A_{0 \mathrm{i}}+A_{1 \mathrm{i}} m$. Thus, in order to explain the inverse reflection from a smooth surface and its subsurface layers, it is natural to assume that in each sub-layer there 

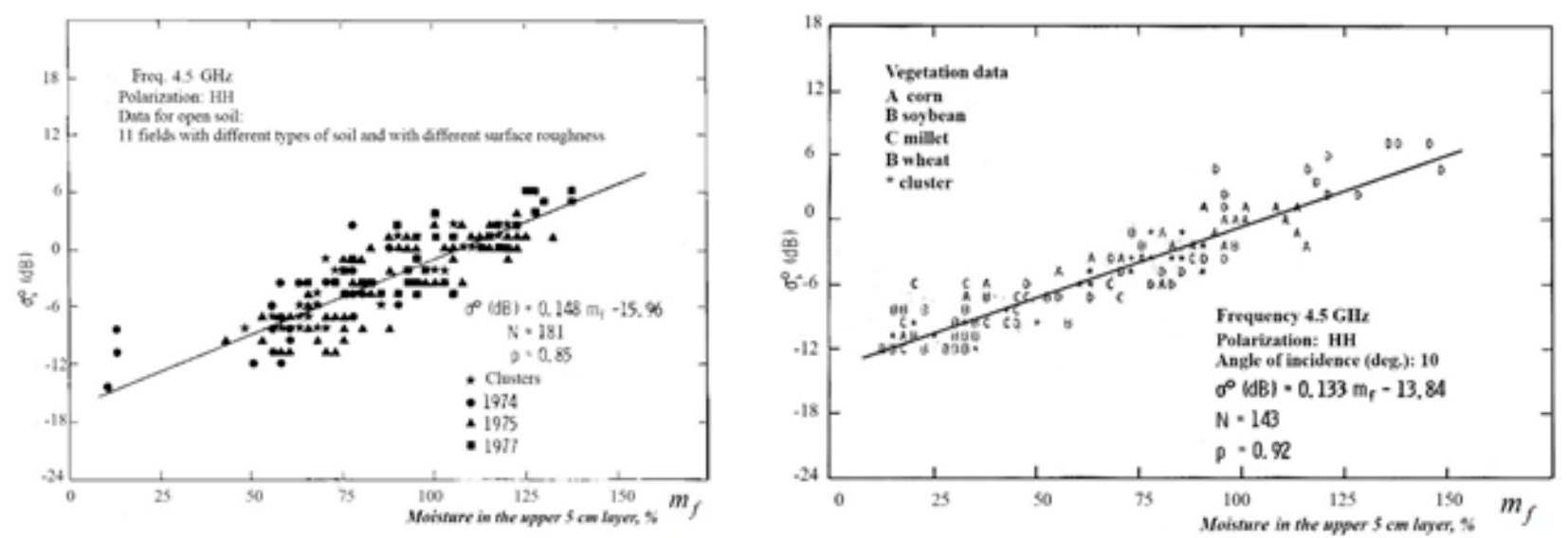

Fig. 10. The coefficient of reverse reflection of the open soil as a function of bumidity (field bumidity) for various conditions of surface roughness and various textural soil compositions [22-24].

are spatial dielectric inhomogeneities within the resolution element. An increase in humidity leads to an increase in the attenuation coefficient $\alpha \sim m$ and simultaneously to a stronger spatial dielectric heterogeneity of the soil, which enhances the inverse reflection.

In formula (1) it is assumed that the reflection coefficients from adjacent layers are independent, i.e. fluctuations in humidity are independent. Calculations of the correlation coefficient between the dependence of humidity on the distance for different layers in our experiment showed that the average value of the moisture coefficient between the layers $0-30 \mathrm{~cm}, 30-83 \mathrm{~cm}, 83-136 \mathrm{~cm}, 136-$ $189 \mathrm{~cm}$ is $0.17-0.15$. Prior to this stage, we only operated on inhomogeneities related to humidity. We extend this representation, generalizing it to all inhomogeneities in general, i.e. we introduce the concept of "effective" humidity. For definiteness, i.e. the construction of a theoretical model, as the investigated soil, we will consider sand with some "effective" moisture.

Thus, the construction of a theoretical model of back reflection will be based on the profiles of "effective" humidity:

1) We represent the soil (of various types, roughness, frozen soil, etc.) in the form of sand, which has some "effective" moisture.

2) We are given an arbitrary depth profile of the "effective" humidity in Fig. $10 a$.

3) Based on the results of $[14,15]$, we determine the dependence of the absorption coefficient of the sub-layers for four wavelength ranges $X=4 \mathrm{~cm}, L=$ $23 \mathrm{~cm}, P=68 \mathrm{~cm}$ and $V H F=254 \mathrm{~cm}$;
4) According to the expressions (3)-(4), we calculate the EPR values for 4 wavelengths;

5) From the ESR values measured as a result of radar survey at the same frequencies with the help of the SAR IMARK complex, we find the integral value of the discrepancy between theoretical (p.4) values and experimental data.

The procedures for clauses 2-5 are repeated until the discrepancies are minimal in Fig. $10 b$.

\section{EXPERIMENTAL RESULTS}

Multi-frequency radar survey was carried out in the winter, which was characterized by heavy snowfalls and strong (up to -30 degrees) frosts. As a priori information was taken - the depth of the snow cover on the fields of $50 \mathrm{~cm}$, the depth of freezing is of the order of $50 \mathrm{~cm}$. Fig. 11 represents the frequency-polarimetric matrix of the radar images of the site (size of a section of $3 \times 3 \mathrm{~km}^{2}$ ) of the selected landfill.

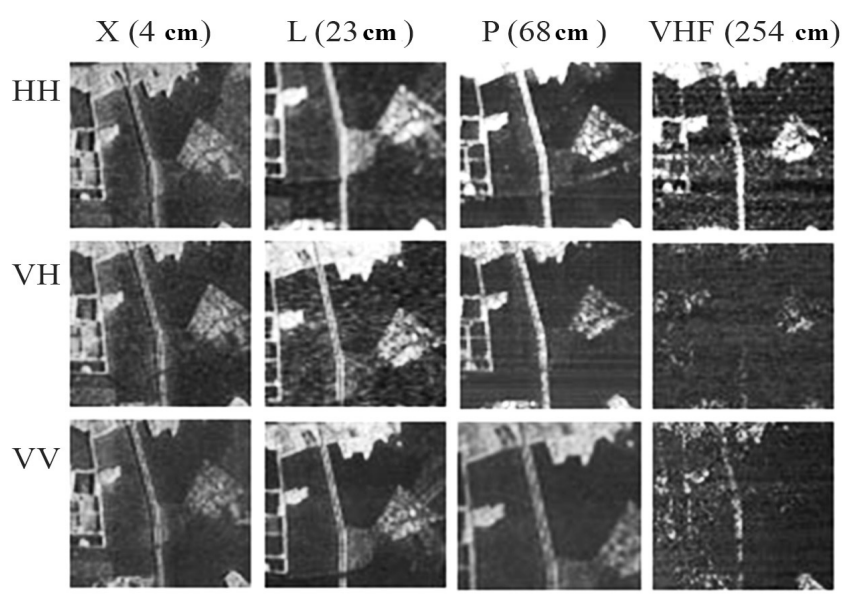

Fig. 11. Multi-frequency Polarimetric Radar Matrix testpolygon. 

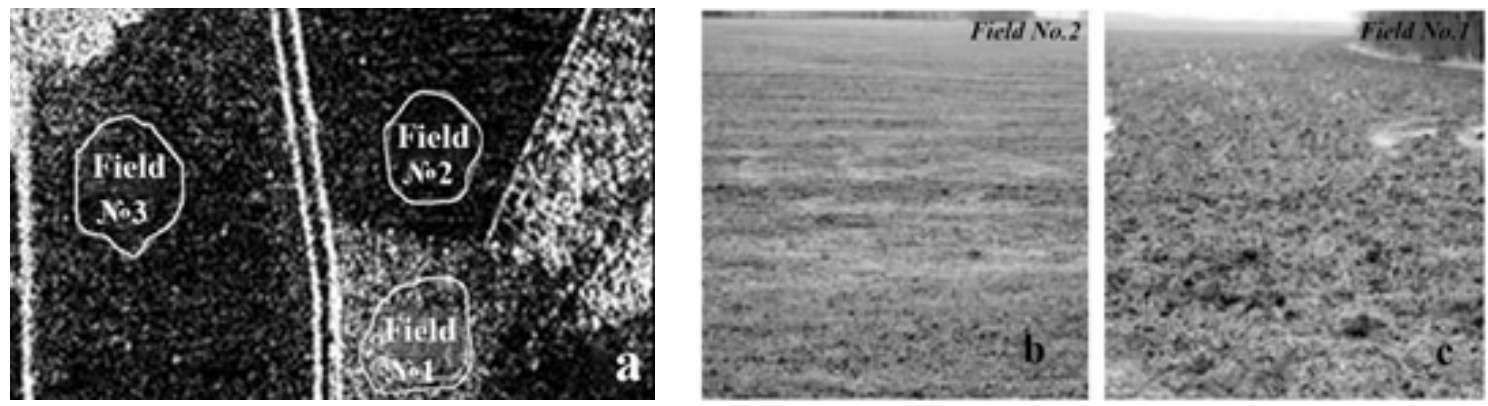

Fig. 12. Areas of the Earth's surface used to interpret the results of RL survey.

Fig. 12 indicates the agricultural fields No. 1, 2, 3, whose RLIs were used to interpret subsurface sensing. Inspection of the fields after the fall of the snow showed: field number No. 2 (Fig. 12b) is a flat area with a shallow roughness and winter wheat shoots; field No.1 (Fig. 12c) is a plowed field with a roughness of about $20-30 \mathrm{~cm}$; field No. 3 is an flat area of terrain with low stubble, in the radar images of which a qualitative difference is observed in the meter range on $V V$ and $H H$ polarizations.

The average intensity and dispersion of the RL signal for field No. 2 are minimal over the entire wavelength range and on all polarizations, so it is chosen as a "support" for relative calibration. Preliminarily, the RLIs of each field was subjected to spatial averaging (spatial filtering) of 2000 pixels to reduce the influence of the spectroscopic structure. After that, three or four identical sections were selected on each field, and there was an average and standard deviation. The values thus obtained were used to plot the contrasts of the wavelengths (Fig. 13). Under performing the work, the radar images in the $X, L$ and $P$ bands were calibrated with corner reflectors, in $V H F$, using reflection from the transmission line supports [18]. Reflection from angle reflectors and power line supports were used to obtain relative contrast values.

From Fig. 13 that the contrasts for different fields and polarizations are different. Therefore, it can be assumed that the effective moisture profile on different polarizations will be different.

\subsection{BuILding an EFFECTIVE moIsture profile FROM DEPTH}

The success of constructing a profile of "effective" humidity depends on the use of available a priori information about the underlying surface [8]. As a priori information was taken:

- The thickness of the snow cover on the selected fields is the same and is $\sim 40-60 \mathrm{~cm}$.

- Between the snow and the soil there is a rough surface layer, which is due to the processing of the land and the presence of vegetation.

- The thickness of the frozen soil in all fields is the same and is $\sim 80 \mathrm{~cm}$.

Fields No. 1 and No. 2 are located in close proximity to each other and therefore it can be assumed that the structure and moisture of the soil at a depth of more than $1.4 \mathrm{~m}$ are the same. The moisture content of the soil (sandy soil), measured at a depth of $\sim 1.5 \mathrm{~m}$ in the vicinity of this field, was $\sim 40 \%$.

In Fig. $14 a$ two proposed moisture profiles on $\mathrm{HH}$ polarization are presented as an example, in Fig. $14 b$ a profile in which the theoretical and experimental contrasts coincide.

The results show that the effective moisture profile depends on the polarization, i.e. different soil layers contribute to the formation of the reverse reflection.
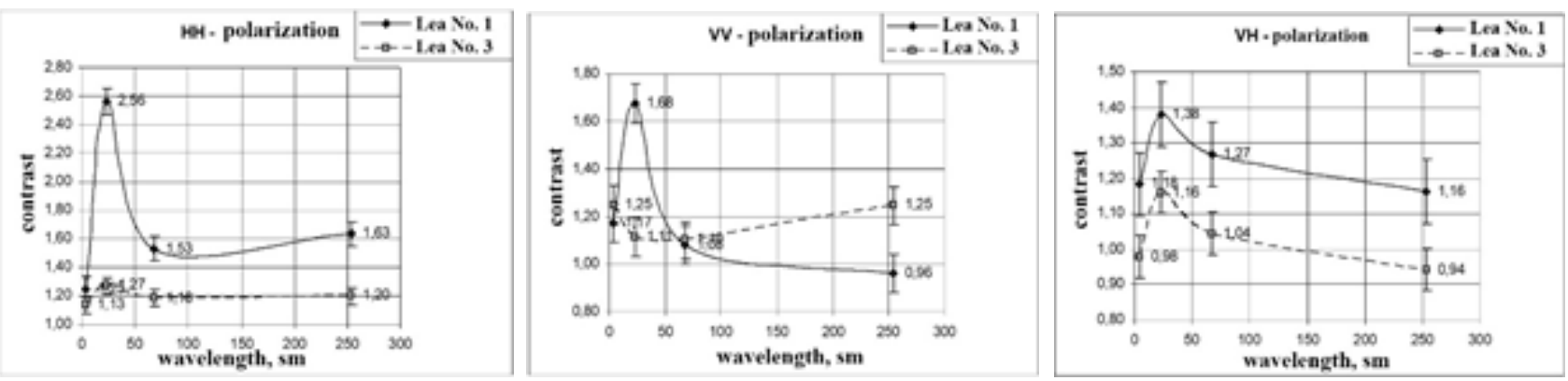

Fig. 13. Relative contrast values for Fields 1 and 3 on different polarizations. 

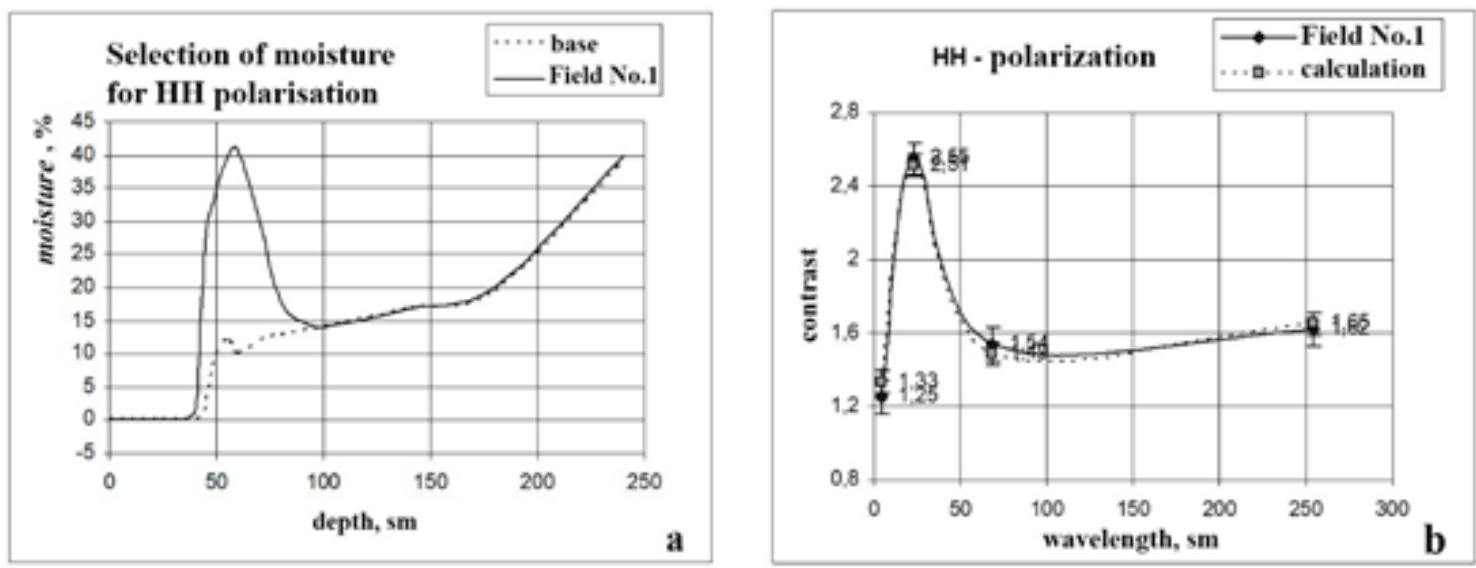

Fig. 14. Profiles of "effective" bumidity on the reference field and field No. 1.

The complexity of the simulation was that there is no information about attenuation in different wavelength ranges, data on the presence of spatial inhomogeneities in snow and in frozen soil on an element of $\sim 15 \times 15 \mathrm{~m}^{2}$. When interpreting it was assumed that the state of the surface (snow, roughness, frozen soil, distribution of humidity along the depth, etc.) corresponds to some effective value of moisture in accordance with the proposed procedure (see paragraphs 4,5 ), the moisture profile was selected, so that the theoretical and experimental contrasts coincide.

\subsection{ANAlysis of the obtained interpretation RESULTS}

When examining $R L$ images, fields No. 1, No. 2, No. 3 were selected (Fig. 12). The analysis shows that for radar fields No. 1 and No. 2 in the $X, P$ and $V H F$ ranges there is no contrast, in the $L$ range the contrast is significant. The conclusion is that, in the $\lambda=4 \mathrm{~cm}$ range, the RLIs are formed by the snow cover, the damping in the snow cover is such that the rough layer of the soil does not affect the reflection in the $X$ range. In the $L$ range, the attenuation in

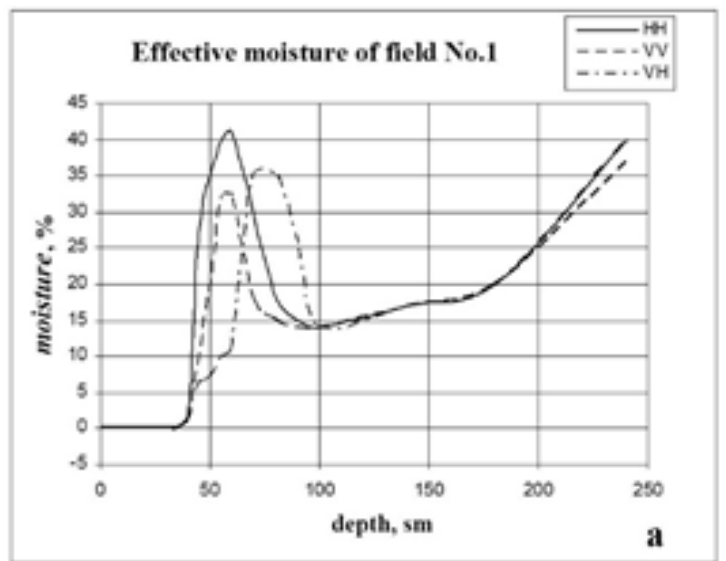

the snow is weakened and the snow-frozen ground affects the character of the image. The heterogeneity of the snow-frozen soil layer for the $P$ and VHF ranges is small and this layer is flat for these ranges. Reflections in the $P$ and VHF bands define deeper layers. The results of the treatment confirm this. At depths of $40-60 \mathrm{~cm}$, a dramatic increase in the effective moisture content is observed, which is determined by the roughness. For $V H$ polarization, this layer turned out to be somewhat deeper. The deep layers affect all polarizations in the same way.

The peculiarity of the obtained results on the determination of the effective moisture profile in field No. 3 is that the transition layer snow-frozen ground has little effect on the inverse reflection in the wavelength bands $L, P$ and VHF. Since the upper soil layer is a stubble, the effect is explainable. For the reverse reflection, the depths are influenced by the depths $h>150 \mathrm{~cm}$. For $V V$ polarization, the effective humidity is greater than the $H H$ polarization, which is manifested in the RLI images (see the element VHF-VV and VHF-HH of the matrix in Fig. 2). A comparison of the photographs

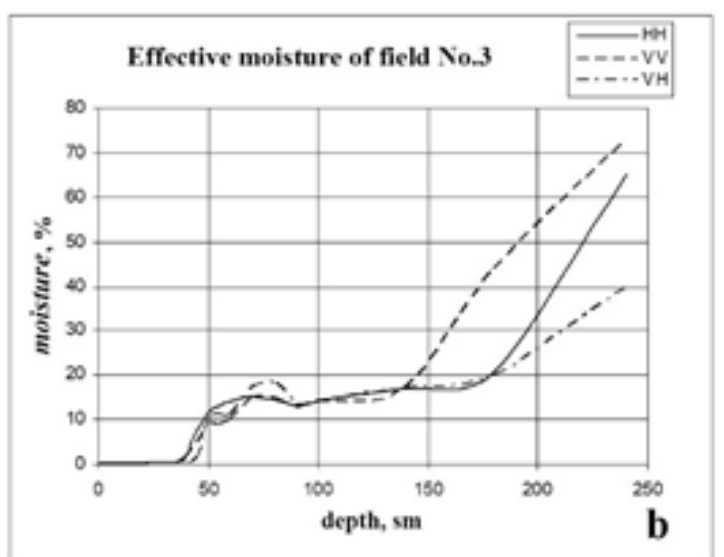

Рис. 15. Profiles of "effective" bumidity in field No. 1 a and field No. 3 b calculated on radar sounding on different polarizations. 
of this area for 2003 and 1987 shows that the area of field No. 3 increases due to the destruction of the forest massif, and the feature in the image is probably explained by the presence of vertical roots under the upper soil layer. Thus, it can be concluded that the proposed method allows analyzing the nature of subsurface soil layers in the winter period of time.

\section{CONCLUSION}

The use of multi-frequency side-view radar with a synthetic aperture for layered subsurface sounding of the earth's surface is of great interest. However, the reconstruction of the deep profile of the upper layer of the Earth's surface by multi-frequency (with a limited number of wavelength ranges) radar images represents an incorrect inverse problem. An approximate solution of the inverse problem in the general case is found by constructing a smoothing functional representing the discrepancy functional between the results of measurements of the scattered field and the model direct problem. For the calculation of a model informative characteristic, a solution of the direct electro-dynamic problem is necessary. However, the physicochemical structure of the upper cover of the earth is so complex that it does not allow to adequately simulate the reverse reflection from subsurface soil layers for different wave bands. The representation of the top cover of the soil in the form of a series of uniform layers with even or rough surfaces is possible only in individual cases. The spatial spectra of the "variegation" of the soil cover both in space and in depth are broad and related both to moisture fluctuations and soil composition. It makes sense to talk only about the averaged layer structure of the soil in a limited area, i.e. to represent the upper layer of soil in a limited area in the form of flat even sub-layers, the reflection of which is due to the "variegated" physicochemical characteristics of this layer and is determined by the Bragg component of the spectrum of "variegation" of this sub-layer.

In order to somehow approach the solution of the problem of layer-by-layer surface sounding of the upper surface cover, unusual postulates have been made in the work, within the framework of which this problem is solved. The upper layer of the soil is supposed to consist of sand, the sub-layers of which have flat surfaces, and all changes in the dielectric constant are related to the "variegation" of the moisture content of the sand, i.e. changes in the type of soil heterogeneity, roughness, salinity, etc., we correlate to the "effective" moisture of sand, which is the main postulate of this work. Given different profiles of "effective" humidity, it is possible to solve a direct problem and construct a smoothing functional that represents the residual functional between the results of measurements of the scattered field and the model direct problem. However, the resulting deep profile of "effective" moisture requires additional physical interpretation, i.e. it is necessary to relate this or that layer of "effective" moisture to a tangible physical value, for example, snow cover, soil roughness under snow cover, frozen soil, etc. This stage of work is performed from purely a priori information, which must correspond to the physical meaning.

The work was carried out with the financial support of the ISTC, project No. 2806.

\section{REFERENCES}

1. "IMARK - Multi-Frequency Airborne Polarimetric Radar System Operating on Board of TU-134A Flying Laboratory for Remote Observations of the Earth's Surface". Moscow Scientific Research Institute for Instrument Engineering, MNIIP/VEGA-M Corporation, Russia, 1998, 8 pp.

2. Freeman A, Durden SL, Zimmerman R. Mapping Subtropical Vegetation using Multi-frequency, Multipolarization SAR Data. Proc.Int. Geoscience and Remote Sensing Symp. 1992:1686-1689.

3. Kutuza B, Shutko A, Plushev V, Ramsey E, Logan B, DeLoach S, Haldin A, Novichikhin E, Sidorov L, Manakov V, Nelson G. Advantages of synchronous multi-spectral SAR and Microwave Radiometric observations of land covers from aircraft platforms. Proc. EUSAR 2000, 3-rd European Conference on SAR (23-25 May 2000), Munich, Germany, 2000:663-666.

4. Ulander L, Pierson W, Lundberg M, Follo P, Frolind P, Gustavsson A. CARABAS-II SAR change detection performance on ground targets concealed in foliage. Proc. of EUSAR 2004, 5th European Conference on Synthetic Aperture Radar, Ulm, GE, 2004:297-300.

5. Gustavsson A, Ulander L. Combining various ISR assets to create a common and complete ground picture. Proc. of EUSAR 2004, 5th 
European Conference on Synthetic Aperture Radar, Ulm, GE, 2004:301-304.

6. Finkelstein M, Karpukhin V, Kutev V, Metelkin V. Podpoverkhnostnaya radiolokatsiya [Subsurface Radiolocation]. Moscow, Radio i svyaz' Publ, 1999, 266 p.

7. Grinev AYu. Voprosy podpoverkhnostnoy radiolokatsi. Moscow, Radiotekhnika Publ, 2006, 416 p.

8. Mirtova E. Resheniye obratnykh zadach podpoverkhnostnoy

optimizatsionnymi metodami [Solution of inverse problems of subsurface radar by optimization methods]. Thesis for the degree of PhD, Riga, 1988, 193 p.

9. Druchinin SV. Models for calculation of dielectric constant of moist sandy-clayey soils in wavelengths from centimeters to tens of meters. Proc. GPR 2000, 8-th Intern. Conference on Ground Penetr. Radar, Gold Coast, Australia, 2000, 26-31.

10. Druchinin SV. Models for calculation of dielectric permittivity of moist sandy-clayey soils in wide range of frequencies. Proc. SSTA 2000, Intern. Conf. on Subsurface Sensing Technologies and Applications, San Diego, (30 July-4 Aug.), California, USA, 2000.

11. Druchinin S, Izyumov S. Measured and calculated dielectric permittivity of moist clayey soils. Proc. 6-th Meeting of Environmental and Engineering Geophysics, Bochum, (Sept. 3-7), Germany, 2000:P-GR09.

12. Kutuza B, Kalinkevich A, Pliushchev V, Druchinin S. Multi-frequency SAR for Object Detection in Upper Subsurface Layers of the Earth. In: Complementary of Ladar and Radar, RTO-MP-092, RTO/NATO, France, 2003.

13. Kalinkevich A, Kutuza B, Pliushchev V. Druchinin S. Zarubę̧haya radioelektronika, Uspekhi sovremennoy radioelektroniki, 2001, 31-39.

14. Leschanskiy J. Lebedeva G. Shumilin V. Elektricheskiye parametry peschanogo i glinistogo gruntov v diapazone santimetrovykh, detsimetrovykh i metrovykh voln. Iqv. VUZov. Radiofizika, 1971, 4(14):563-569.

15. Druchinin S. Models for calculation of dielectric constant of moist sandy-clayey soils in wavelengths from centimeters to tens of meters. Proc. 8th Intern. Conf. on Ground Penetrating Radar, (27 April 2000), doi: 10.1117/12.383576.
16. Mirtova EV, Karpukhin VI. Issledovaniye primeneniya optimal'nykh metodov $\mathrm{k}$ resheniyu obratnykh zadach zondirovaniya ledovykh pokrovov. Teoriya i tekhnika radiolokatsii, navigatsii $i$ radiosyyazi v GA, Riga, 1986:38-44.

17. Druchinin S, Izyumov S. Measured and calculated dielectric permittivity of moist clayey soils. Proc. 6-th Meeting of Environmental and Engineering Geophysics, Bochum, (Sept. 3-7), Germany, 2000:P-GR09.

18. Kalinkevich AA, Krilova MS, Manakov VYu. K voprosu ob ispol'zovanii opor LEP v kachestve repernykh ob'yektov pri interpretatsii RLI RSA UKV diapazona. Trudy 4 V seross. nauchn. konf. "Radiofizicheskiye metody distantsionnogo zondirovanii sred", Murom, 30.06.-3.07.2009, 186-190.

19. Grinev AYu, Chislennyye metody resheniya prikladnykh zadach elektrodinamiki [Numerical methods for solving applied problems of electrodynamics], Moscow, Radiotekhnika Publ, 2012, 336 p.

20. Yakovlev O, Yakubov V, Uryadov V. Pavel'yev A. Rasprostraneniye radiovoln [Propagation of radio waves]. Moscow, Lenand Publ., 2009, 496 p.

21. Bass F, Fuks I. Rasseyaniye voln na statisticheski nerounoy poverkhnosti [Scattering of waves on a statistically irregular surface], Moscow, Nauka Publ, 1972, 424 p.

22. Ulaby FT, Moore RK, Fung AK. Microwave remote sensing. London, Addison-Wes1 ey Public. Comp., 1981.

23. Ulaby FT, Batlivala PP. Optimum Radar Parameters for Mapping Soil Moisture. IEEE Transaction and Geoscience Electronics, 1976, 2(GE-14):81-93.

24. Ulaby FT, Cihlar J. Moore RK. Active Microwave Measurement of Soil Water content. Remote Sensing of Environment, 1974, 3:185-203.

25. Chubarov V. Pitaniye gruntovykh vod peschanoy pustyni cherez, zonu aeratsii [Groundwater supply of sandy desert through aeration zone]. Moscow, Nedra Publ., 1972, 135 p.

26. Marchuk V, Smirnov V. Trudy V seross. nauchn. konf. "Sverkhshirokopolosnyye signaly v radiolokatsii, svyazi i akustike", Murom, 2003:455-459.

27. Marchuk V. Metodiki provedeniya eksperimentov po radiolokatsionnomu podpoverkhnostnomu zondirovaniyu Zemli i planet zemnoy gruppy [Methods of conducting experiments on radar subsurface probing of the Earth and the 
terrestrial planets]. Thesis for the degree of $\mathrm{PhD}$. Fryazino, 2008, 163 p.

28. Marple SL. Digital Spectral Analysiswith Applications. Prentice-Hall, Englewood Cliffs, NJ, 1987.

29. Armand N, Lukin D, Chubinskiy N. Nauchnyy sovet Rossiyskoy Akademii nauk po kompleksnoy probleme "Rasprostraneniye radiovoln", Muromskiy institut Vlad.GU.

30. Kalinkevich A, Krylova M, Masyuk V, Marchuk V. Using georadars to study heterogeneities of the top layer of coniferous forest soil. Radiotekhnika, 2009, 3:98-103.

31. Tikhonov A, Arsenin V. Metody resheniya nekorrektnykh zadach [Methods for solving illposed problems]. Moscow, Nauka Publ., 1979, $285 \mathrm{p}$.

32. Khilkevich V. Opredelenie parametrov zemnykh pokrovov s pomoshchyu neyronnogo metoda. Trudy V seros nauchn. konf. "Distantsionnoye zondirovaniye zemnykh pokrovov $i$ atmosfery aerokosmicheskimi sredstvami", (June 20-22) Murom, 2001:147-151. 